Artigo original

\author{
Fernando Diefenthaeler ${ }^{1}$ \\ Rodrigo Rico Bini ${ }^{1}$ \\ Ana Paula Barcellos Karolczak ${ }^{1,2}$ \\ Felipe Pivetta Carpes ${ }^{1}$
}

\title{
ATIVAÇÃO MUSCULAR DURANTE A PEDALADA EM DIFERENTES POSIÇÕES DO SELIM
}

\section{MUSCLE ACTIVATION DURING PEDALING IN DIFFERENT SADDLE POSITION}

\begin{abstract}
RESUMO
O objetivo do presente estudo foi analisar os efeitos de diferentes posicionamentos do selim no padrão de ativação elétrica durante a pedalada. Três ciclistas de elite foram submetidos a um protocolo que constou da avaliação de quatro diferentes posições de selim (para frente, para trás, para cima e para baixo), assumindo como posição de referência a posição usada durante os treino e competição. O deslocamento do selim foi de $1 \mathrm{~cm}$ para todas as posições. As bicicletas dos ciclistas foram acopladas a um ciclossimulador magnético. A carga do teste foi normalizada utilizando um critério fisiológico, garantindo assim o mesmo nível de esforço para todos os sujeitos. A atividade elétrica de seis músculos do membro inferior foi mensurada: gluteus maximus, rectus femoris, biceps femoris, vastus lateralis, gastrocnemius medialis, e tibialis anterior. Os resultados demonstram que pequenos ajustes na posição do selim podem afetar os padrões de ativação elétrica e provavelmente a técnica de pedalada.
\end{abstract}

Palavras-chave: Ciclismo; Eletromiografia; Ativação muscular.

\section{ABSTRACT}

The purpose of this study was to analyze the effects on muscle activation pattern of different saddle positions during cycling. Three elite cyclists followed a protocol that consisted of four different saddles positions, displaced forward, backward, upward and downward with relation to the reference position used in training and competition. In all tests the saddle was displaced by $1 \mathrm{~cm}$. The cyclists' bicycles were mounted on a magnetic cycle simulator. The load was normalized at the second ventilatory threshold. Muscle activation of six lower limb muscles was registered: gluteus maximus, rectus femoris, biceps femoris, vastus lateralis, gastrocnemius medialis, and tibialis anterior. The results demonstrated that relatively small saddle adjustments can affect the pattern of muscle activation and probably cycling technique.

Key words: Cycling; Electromyography; Muscular activity.

1 Laboratório de Pesquisa do Exercício, Escola de Educação Física da Universidade Federal do Rio Grande do Sul. Brasil 


\section{INTRODUÇÃO}

Pesquisas em biomecânica visando à otimização do desempenho de ciclistas tem sido o objetivo de vários pesquisadores nos últimos anos. Além do estudo das forças envolvidas na pedalada ${ }^{1,2}$, podem ser analisados os padrões de ativação muscular por meio da eletromiografia (EMG) e, desta forma, compreender qual a contribuição de cada músculo envolvido no movimento da pedalada, bem como as alterações decorrentes dos diferentes ajustes adotados pelo ciclista ${ }^{3}$.

A eletromiografia de superfície (EMG) fornece informações sobre o padrão de ativação muscular e sobre como o sistema nervoso central controla o movimento. Essa técnica tem sido amplamente utilizada para estudar a atividade muscular e a coordenação neuromuscular no ciclismo ${ }^{3,4-6}$. Comumente, no estudo da pedalada, os padrões de atividade muscular são descritos em relação ao ângulo do pé-de-vela, no qual, em geral, a maior ativação dos músculos ocorre durante a fase propulsiva $\left(0-180^{\circ}\right)$, quando grande parte da energia necessária para pedalar é transferida para o pé-de-vela ${ }^{3}$.

Os músculos monoarticulares extensores das articulações do quadril, do joelho e do tornozelo (gluteus maximus - GM, soleus - SOL, vastus lateralis - VL e vastus medialis - VM) apresentam uma grande ativação durante a fase de propulsão; e o tibialis anterior (TA), durante a fase de recuperação da pedalada (180 $-360^{\circ}$ ). Já os músculos do grupo posterior da coxa apresentam maior atividade entre 90 e $135^{\circ}$ do ciclo da pedalada; enquanto que o rectus femoris (RF) apresenta ativação tanto na fase de propulsão, como um extensor do joelho, como na fase de recuperação, agindo como flexor do quadril ${ }^{3}$.

Segundo Gregor et al. ${ }^{7}$, o GM e o biceps femoris (BF) atuam no torque extensor do quadril entre 0 e $180^{\circ}$ do ciclo da pedalada; o RF, o VM e o VL parecem ter maior atuação no torque extensor do joelho entre 0 e $75^{\circ}$; e o semimembranaceus (SM), o BF, o gastrocnemius medialis (GAM) e o gastrocnemius lateralis (GAL) parecem desempenhar uma importante função no torque flexor do joelho, observado a $180^{\circ}$ do ciclo da pedalada. Sendo assim, diversos autores, buscam por meio da análise da ativação eletromiográfica dos músculos envolvidos no movimento da pedalada, entender as diferentes estratégias neuromusculares adotadas pelos ciclistas ${ }^{6,8-11}$.

Os padrões de atividade muscular durante a pedalada dependem dos diferentes ajustes da bicicleta (altura do selim, tamanho do pé-de-vela, tamanho do quadro, etc.), da posição adotada pelo ciclista, da relação de marchas e da técnica da pedalada ${ }^{3}$. A carga de trabalho e a cadência da pedalada também têm influência direta na atividade muscular ${ }^{6,12,13}$.

Entre os ajustes de posicionamento freqüentemente realizados pelos ciclistas, que podem alterar o padrão de ativação muscular, está a regulagem da altura do selim. As alterações na ativação elétrica ocorrem em detrimento da mudança do ângulo ótimo de produção de força dos músculos envolvidos no movimento da pedalada ${ }^{3}$. Até o momento, os estudos realizados com EMG em diferentes alturas do selim têm demonstrado resultados variados. Algumas pesquisas apontam um aumento na ativação muscular em função da diminuição da altura do selim, especialmente para os músculos isquiotibiais e quadríceps ${ }^{3,9,10}$.

No entanto, os resultados do estudo de Ericson et al. ${ }^{12}$ apontam uma maior ativação elétrica dos músculos GM, SM, SOL e GAM com o aumento da altura do selim; enquanto que, no estudo de Houtz e Fischer ${ }^{8}$, os resultados da EMG apontam uma menor ativação ao pedalar-se com selim em uma posição mais elevada para os mesmos músculos.

A necessidade de se estudar a ativação muscular ao variar-se a posição do ciclista na bicicleta tem uma importância fundamental no momento da escolha do tamanho do quadro da bicicleta, do tamanho do pé-devela a ser adotado, da regulagem da altura do selim, do tamanho do avanço e dos demais ajustes do complexo ciclista-bicicleta.

Todas essas alterações afetarão diretamente o desempenho do atleta; no entanto, essa é uma questão difícil de ser generalizada, e talvez existam ajustes relativamente pequenos que podem exercer grande influência nas questões levantadas acima, de maneira distinta para diferentes ciclistas. Portanto, investigar essa questão de forma individualizada torna-se essencial em se tratando de ciclistas de alto nível. Sendo assim, o objetivo do presente estudo foi descrever as respostas individuais no período de ativação muscular, em função de mudanças nas posições vertical e horizontal do selim de ciclistas de elite.

\section{PROCEDIMENTOS METODOLÓGICOS}

\section{Sujeitos}

Participaram deste estudo três atletas selecionados intencionalmente entre os 10 melhores ciclistas do ranking da Federação Gaúcha de Ciclismo, com

Tabela 1. Caracterização dos ciclistas, com a idade, tempo de treinamento, volume semanal de treino e os principais títulos individuais.

\begin{tabular}{ccccc}
\hline Ciclista & $\begin{array}{c}\text { Idade } \\
(\text { anos })\end{array}$ & $\begin{array}{c}\text { Tempo de treinamento } \\
(\text { anos })\end{array}$ & $\begin{array}{c}\text { Volume de treino } \\
(\mathrm{km} / \text { semana) }\end{array}$ & Principal título \\
\hline A & 30 & 18 & 450 & Tri-campeão Gaúcho da categoria elite \\
B & 23 & 4 & 600 & Campeão Gaúcho categoria sub-30 \\
C & 24 & 8 & 450 & Campeão Gaúcho categoria sub-30 \\
\hline
\end{tabular}


diferentes níveis de experiência, conforme a Tabela 1 que fornece as características individuais e um breve histórico competitivo dos ciclistas avaliados. Todos assinaram um termo de consentimento concordando com sua participação voluntária na pesquisa. Este projeto foi aprovado pelo Comitê de Ética em Pesquisa com Seres Humanos da Universidade Federal do Rio Grande do Sul (protocolo n. 2004311).

\section{Aquisição dos dados}

Eletromiografia de superfície - Foi avaliada a atividade elétrica de seis músculos: (1) GM; (2) RF; (3) VL; (4) BF; (5) TA; e (6) GAM do membro inferior direito dos ciclistas. Para a aquisição dos dados, foi utilizado um eletromiógrafo de oito canais (Bortec Eletronics Inc., Calgary, Canadá). Eletrodos de superfície passivos (Kendal Meditrace, $\mathrm{Ag} / \mathrm{AgCl}$, com diâmetro de 2,2 cm), colocados em configuração bipolar, foram fixados sobre o ventre dos músculos, alinhados de acordo com a orientação das fibras musculares; e um eletrodo de referência (terra) foi fixado na face anterior da tíbia.

Anteriormente à colocação dos eletrodos, a impedância elétrica da pele foi reduzida ${ }^{14}$, mediante a tricotomia e a limpeza da pele com álcool, a fim de remover as células mortas e o oleosidade da pele no local do posicionamento dos eletrodos. A seguir, os eletrodos foram fixados na pele, e uma leve pressão manual foi aplicada sobre eles para aumentar o contato entre o gel do eletrodo e a pele ${ }^{15}$. As técnicas de preparação e aplicação dos eletrodos na pele estão de acordo com as recomendadas pela SENIAM ${ }^{16}$. Concluídas as etapas de fixação dos eletrodos e posicionamento dos cabos, os ganhos no eletromiógrafo foram ajustados para cada um dos músculos.

Após os sinais eletromiográficos terem sido amplificados, os mesmos passaram por um conversor analógico-digital de 16 canais do sistema CODAS (Dataq Instruments, Inc. Akron, USA). Para análise e processamento dos sinais EMG, foi utilizado o sistema SAD (Laboratório de Medições Mecânicas, Escola de Engenharia, UFRGS, www.ufrgs.br/lmm). A freqüência de aquisição dos dados da EMG foi de $2.300 \mathrm{~Hz}$ por canal.

Cinemetria - Simultaneamente a análise da atividade elétrica muscular, o padrão angular de movimento do membro inferior foi analisado bidimensionalmente. Para isso, foi utilizado um sistema de videografia bidimensional Peak Motus (Peak Performance, Inc., USA). Uma câmera, operando a uma freqüência de amostragem de $180 \mathrm{~Hz}$, foi posicionada perpendicularmente ao plano de movimento, a uma distância de aproximadamente $4 \mathrm{~m}$, o que permitiu a aquisição de imagens adequadas para o cálculo das variáveis de interesse deste estudo.

A identificação dos pontos anatômicos de referência que permitiram a determinação das variáveis cinemáticas no plano sagital foi feita com marcadores reflexivos colados sobre a pele e na sapatilha direita dos ciclistas. Os pontos de referência foram digitalizados automaticamente por meio do sistema de cinemetria empregado neste estudo, com uma incerteza estimada em $0,05 \%$ pelo fabricante. Os pontos anatômicos de referência utilizados para o cálculo das variáveis cinemáticas de interesse foram: (1) acrômio direto; (2) trocânter maior direito; (3) epicôndilo lateral do joelho direito; (4) maléolo lateral direito; (5) calcâneo; (6) IV metatarso. Calcâneo e IV metatarso foram marcados sob a sapatilha do atleta.

A partir da análise trigonométrica dos pontos mencionados acima, foi possível determinar as variáveis angulares dos segmentos de interesse. As variáveis angulares neste estudo foram: (1) o ângulo do tronco, definido como o ângulo formado entre o eixo horizontal e o segmento de reta formado pelos pontos anatômicos de referência do trocânter maior e do acrômio direito; (2) o ângulo do quadril, definido como o ângulo formado entre o eixo horizontal e o segmento de reta formado pelos pontos anatômicos de referência do trocânter maior e do epicôndilo lateral direito; (3) o ângulo do joelho, definido como o ângulo relativo entre a coxa (representada pelo segmento de reta formado pelos pontos anatômicos de referência do trocânter maior e do epicôndilo lateral direito) e a perna (representada pelo segmento de reta formado pelos pontos anatômicos de referência do epicôndilo lateral e do maléolo lateral direito); e (4) o ângulo do tornozelo, definido como o ângulo relativo entre a perna (representada pelo segmento de reta formado pelos pontos anatômicos de referência do epicôndilo lateral e do maléolo lateral direito) e o pé (representado pelo segmento de reta formado pelos pontos anatômicos de referência do calcâneo e do IV metatarso), conforme Figura 1. Todos os movimentos foram avaliados no plano sagital considerando apenas um grau de movimento (flexão/extensão).

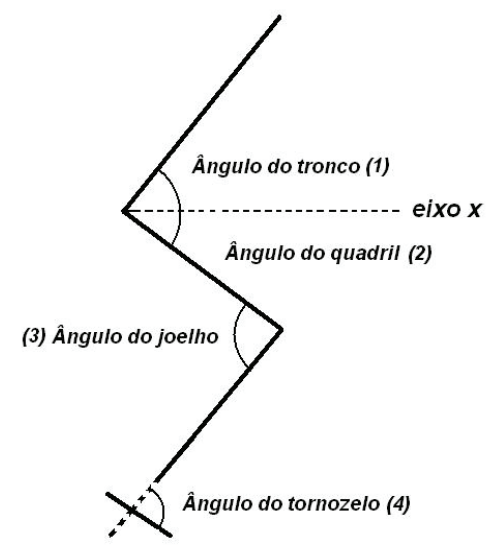

Figura 1 - Ângulos articulares entre os segmentos do tronco, da coxa, da perna e do pé.

Protocolo - Os ciclistas realizaram o protocolo de teste utilizando a suas próprias bicicletas acopladas a um ciclossimulador magnético Cateye CS1000 (Cateye CO., Osaka, Japão), com ajuste de carga em Watts e inclinação em graus. A carga do teste foi normalizada por um critério fisiológico (segundo limiar ventilatório), 
simulando o ritmo de prova dos ciclistas 2,17 . O consumo de oxigênio $\left(\mathrm{VO}_{2}\right)$ e a produção de dióxido de carbono $\left(\mathrm{VCO}_{2}\right)$ foram obtidos por meio de espirometria de circuito aberto por analisador de gases do modelo CPX/D (Medical Graphics Corp., St. Louis, EUA).

A partir da posição do selim adotada pelo ciclista, ou seja, aquela em que o atleta está habitualmente adaptado para treinamentos e competições, denominada neste estudo como posição de referência, quatro novas posições para o selim foram ajustadas durante o protocolo: (1) $1 \mathrm{~cm}$ para cima; (2) $1 \mathrm{~cm}$ para baixo; (3) $1 \mathrm{~cm}$ para frente; e (4) $1 \mathrm{~cm}$ para trás, para os três ciclistas, com exceção do ciclista $B$, que não realizou o teste na posição com o selim para trás.

A partir da primeira posição avaliada para todos, que foi com o selim na posição de referência, a ordem de alteração da posição do selim foi aleatória para cada indivíduo. A posição de referência foi repetida no final do teste para verificar a possibilidade de instalação de mecanismos fadiga devido à realização do protocolo. As mudanças de posição foram feitas logo após o término da coleta da posição anterior. Foi solicitado que o ciclista ficasse em pé sobre os pedais, sem que fosse necessário descer da bicicleta. O tempo de troca entre as posições foi padronizado em $30 \mathrm{~s}$. Durante a execução do protocolo, o atleta manteve constante a sua cadência de pedalada preferida assim como a relação de marchas.

\section{Análise dos dados}

Sinais eletromiográficos - Foi utilizado um filtro digital do tipo Butterworth de quinta ordem, com freqüência de corte entre 10 e $500 \mathrm{~Hz}$. Para o cálculo do envoltório Root Mean Square (RMS), foi utilizado um janelamento móvel do tipo Hamming com janelas de 40 $\mathrm{ms}$, o qual permitiu mensurar a magnitude da ativação muscular bem como os períodos de ativação $0^{18}$. O envoltório RMS foi normalizado e expresso em valores percentuais; e, como critério de normalização, foi adotado o valor do pico máximo do envoltório $\mathrm{RMS}^{19} \mathrm{de}$ cada um dos seis músculos durante o teste, na posição de referência, conforme ilustra a Figura 2.

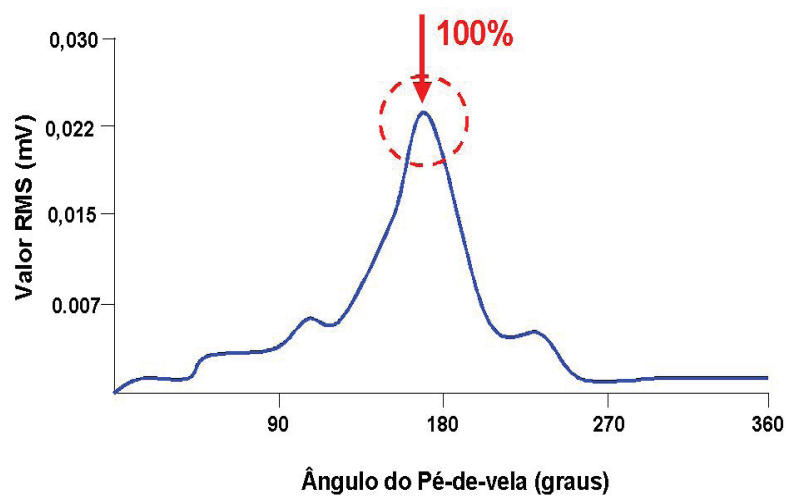

Figura 2. Critério utilizado para determinar o pico máximo do envoltório RMS de cada músculo na posição de referência.

A determinação do valor usado como referência para definição dos períodos de ativação e repouso muscular ao longo do ciclo da pedalada foi baseada na proposta de Baum e $\mathrm{Li}^{6}$, a qual preconiza que o músculo encontra-se ativado quando atinge o limiar correspondente a $10 \%$ do seu valor máximo (Figura 3 ).

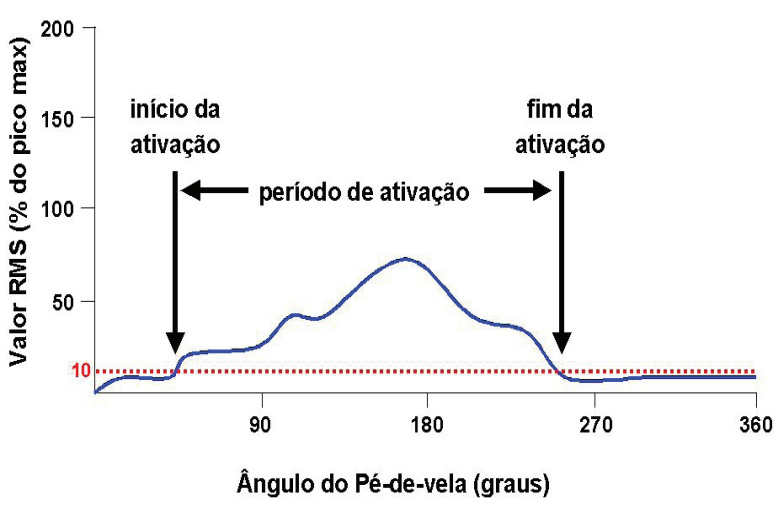

Figura 3. Critério utilizado para demarcação do período de ativação dos músculos.

Cinemetria - Após a digitalização das imagens, os dados foram tratados e submetidos a um processo de filtragem para remoção de ruídos. Os dados angulares foram filtrados por meio de um filtro digital do tipo Butterworth de segunda ordem com freqüência de corte de $5 \mathrm{~Hz}$.

Foram analisados os sinais eletromiográficos e as variáveis cinemáticas correspondentes à média de 10 ciclos de pedalada registrados em cada uma das cinco posições do selim avaliadas. Para que as variáveis supracitadas pudessem ser analisadas, cada ciclista permaneceu durante 3 minutos em cada posição do selim, a partir do momento da estabilização da taxa de troca respiratória (RER) e do $\mathrm{VO}_{2}$ na carga correspondente à intensidade preconizada, e os dados foram coletados nos últimos $30 \mathrm{~s}$. Para que os sinais pudessem ser expressos em função do ângulo do pé-de-vela, foi utilizado um sensor eletromagnético, do tipo reed switch, para a determinação do início e do fim de cada ciclo da pedalada ${ }^{13}$. Os sistemas de EMG e cinemetria foram sincronizados, permitindo que a posterior análise dos dados fosse feita de forma simultânea.

Análise estatística - realizou-se a análise dos dados por meio de estatística descritiva, com o objetivo de descrever as respostas individuais de ciclistas de elite em relação a mudanças na configuração de sua bicicleta. Não se realizou estatística inferencial com o objetivo de evitar a comparação entre os sujeitos.

\section{RESULTADOS}

Os resultados do presente estudo: o (1) taxa de troca respiratória (RER); a (2) ativação muscular (EMG); e (3) os dados cinemáticos são apresentados de forma descritiva.

Durante o teste, os ciclistas pedalaram em uma 
Tabela 2. Valores do consumo de oxigênio dos ciclistas A, B e C em todas as posições avaliadas e as respectivas médias e desvios-padrão.

\begin{tabular}{|c|c|c|c|c|c|c|c|}
\hline \multirow[t]{2}{*}{ Ciclista } & \multicolumn{7}{|c|}{$\begin{array}{l}\mathrm{VO}_{2}\left(\mathrm{ml} \cdot \mathrm{kg}^{-1} \cdot \mathrm{min}^{-1}\right) \\
\text { Posição do selim }\end{array}$} \\
\hline & Referência & Baixo & Cima & Frente & Trás & Média & DP \\
\hline$A$ & 57,2 & 58,6 & 58,0 & 57,9 & 58,9 & 58,0 & 0,51 \\
\hline B & 56,9 & 56,2 & 56,9 & 58,2 & - & 57,1 & 0,86 \\
\hline C & 64,1 & 65,8 & 65,5 & 65,8 & 64,5 & 65,2 & 0,79 \\
\hline
\end{tabular}
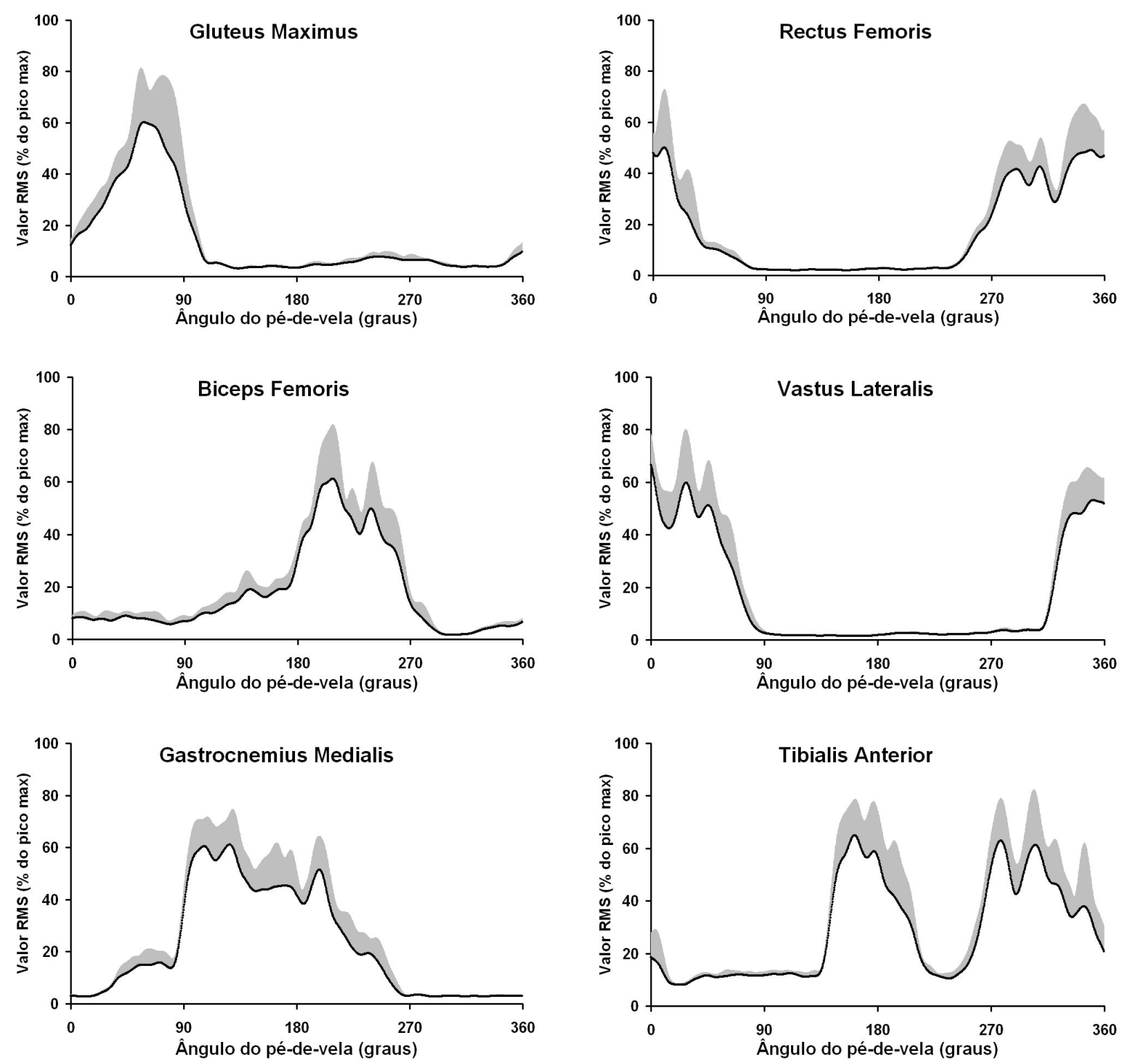

Figura 4. Média e desvio-padrão (valores positivos) do valor RMS normalizado dos músculos GM, RF, BF, VL, GAM e TA durante 10 ciclos de pedalada na posição de referência do ciclista $A$.

intensidade normalizada pelo RER entre 0,90 e 1, que representa uma intensidade muito próxima do limiar anaeróbio individual ${ }^{20,21}$. O ciclista A apresentou RER médio de 0,94 $\pm 0,03$; enquanto que os ciclistas B e C apresentaram médias de 0,94 $\pm 0,04$ e 0,98 \pm 0,04 , respectivamente. $\mathrm{O}$ consumo de oxigênio $\left(\mathrm{VO}_{2}\right)$ manteve-se com baixa variabilidade e em todas as posições avaliadas para os três ciclistas, conforme a
Tabela 2, sendo que a diferença do $\mathrm{VO}_{2}$ não excedeu o valor de $1,8 \mathrm{ml} \cdot \mathrm{kg}^{-1} \cdot \mathrm{min}^{-1}$, não implicando, dessa forma, em mudanças no custo metabólico ${ }^{22}$.

Conforme reportado, o movimento da pedalada é dividido em fases de propulsão $\left(0-180^{\circ}\right)$ e de recuperação $\left(180-270^{\circ}\right)$; porém, para facilitar a discussão dos resultados dos períodos de ativação obtidos no presente estudo, o ciclo foi subdividido em 
quatro quadrantes. No primeiro quadrante $\left(0-90^{\circ}\right)$, observa-se atividade do GM como extensor do quadril, e do RF e do VL como extensores do joelho. No segundo quadrante $\left(90-180^{\circ}\right)$, ocorre ativação do GAM atuando na flexão plantar, e do BF atuando na flexão do joelho. No terceiro quadrante $\left(180-270^{\circ}\right)$, o BF e o GAM apresentam ativação como flexores do joelho; e o TA, como flexor dorsal. Já no quarto quadrante, observa-se ativação do TA, do RF (atuando agora como flexor do quadril) e, por último, do VL, no final desse quadrante, realizando extensão do joelho.

A título de exemplificar como foram realizados os cálculos dos períodos de ativação muscular, ao longo

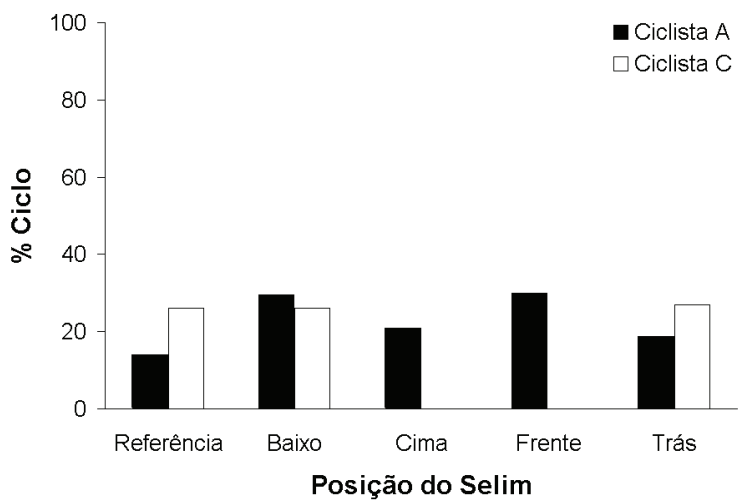

Figura 5.1. Período de ativação do músculo Gluteus Maximus (GM) durante o ciclo de pedalada dos ciclistas A e C.

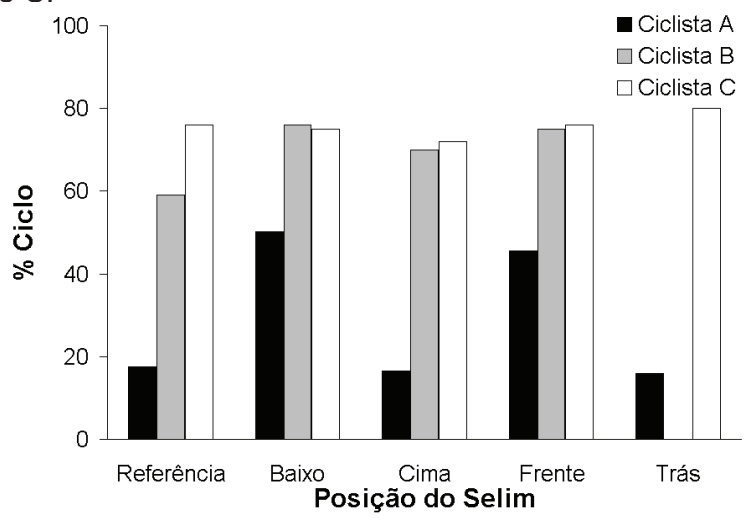

Figura 5.3. Período de ativação do músculo Biceps Femoris (BF) durante o ciclo de pedalada dos ciclistas $\mathrm{A}$, $\mathrm{B}$ e C.

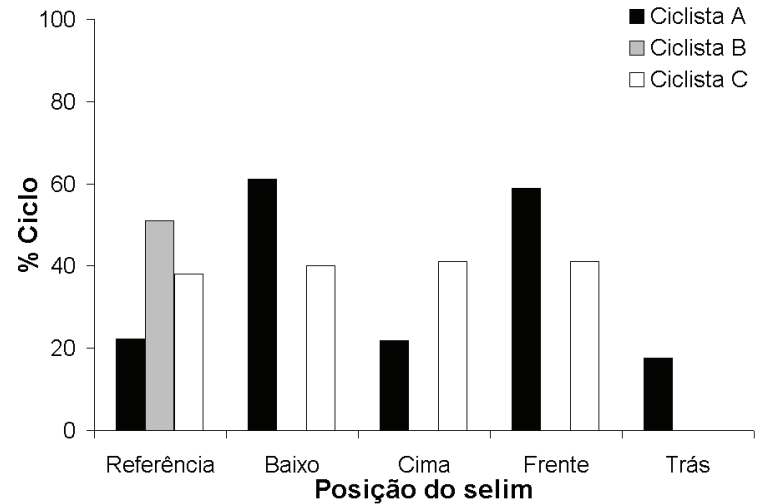

Figura 5.5. Período de ativação do músculo Gastrocnemius Medialis (GAM) durante o ciclo de pedalada dos ciclistas A, B e C. do ciclo e em cada quadrante, utilizando o critério dos $10 \%$ do pico máximo, o valor RMS é expresso em função da posição do pé-de-vela dos seis músculos avaliados do ciclista $A$, na posição de referência, estão demonstrados na Figura 4.

Os resultados da EMG são apresentados em função do período de ativação total ao longo do ciclo de pedalada normalizado, conforme Figura 5. Os resultados gráficos serão apresentados simultaneamente para todos os ciclistas avaliados nas cinco posições do selim; porém alguns músculos dos ciclistas $\mathrm{B}$ e $\mathrm{C}$ não são apresentados devido a limitações técnicas durante a coleta de dados. Foi considerado como critério de

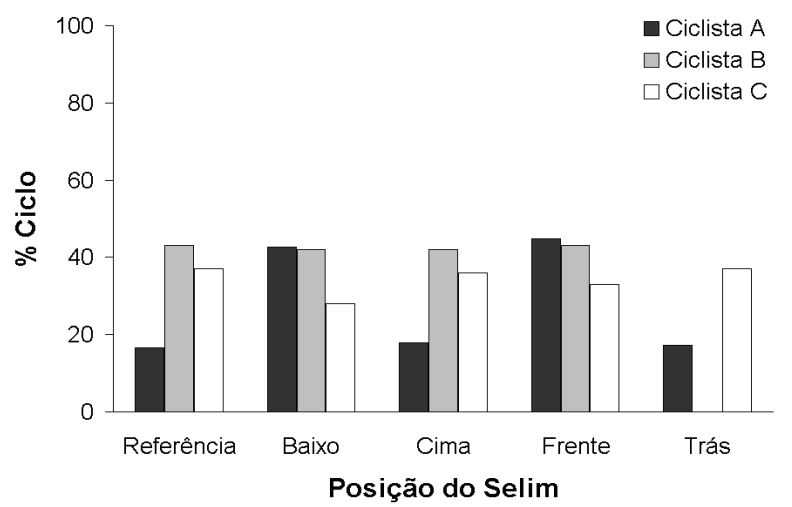

Figura 5.2. Período de ativação do músculo Rectus Femoris (RF) durante o ciclo de pedalada dos ciclistas A, B e C.

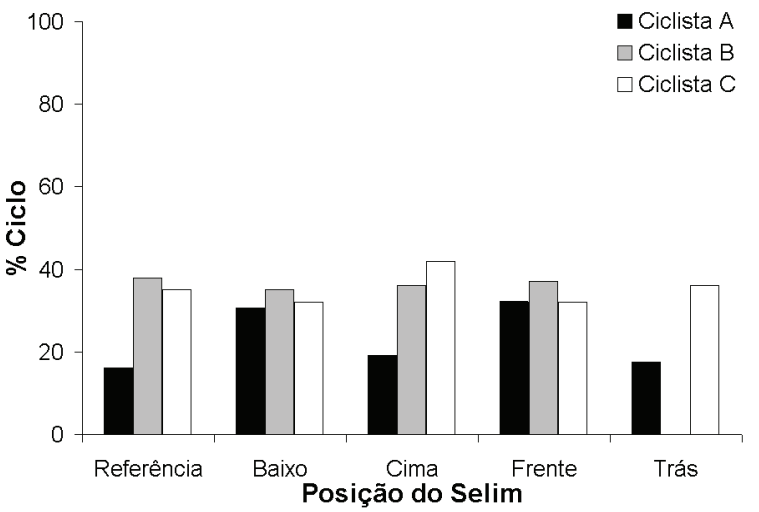

Figura 5.4. Período de ativação do músculo Vastus Lateralis (VL) durante o ciclo de pedalada dos ciclistas A, $\mathrm{B}$ e C.

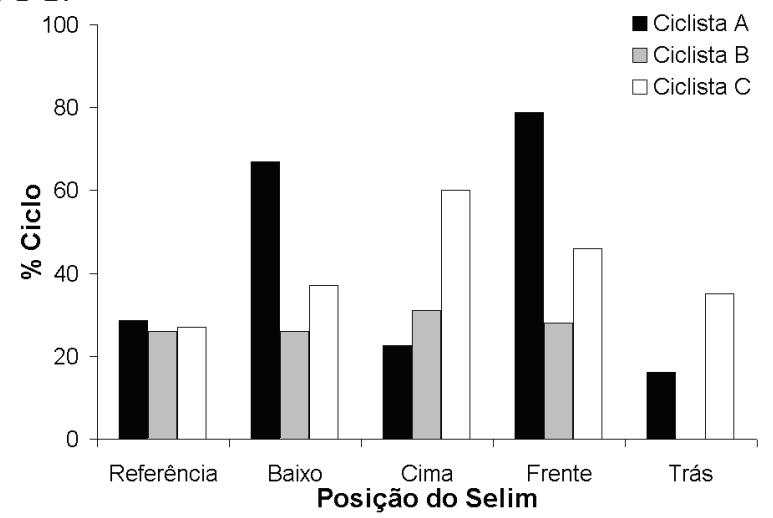

Figura 5.6. Período de ativação do músculo Tibialis Anterior (TA) durante o ciclo de pedalada dos ciclistas A, B e C. 
aumento ou redução no período de ativação valores iguais ou superiores a $5 \%$ em relação à posição de referência individual.

O GM do ciclista A apresentou um aumento no período de ativação em todas as posições em relação à posição de referência (para baixo $15 \%$; para cima $7 \%$; para frente $16 \%$ e para trás $5 \%$ ); enquanto que no ciclista $C$ não alterou (Figura 5.1). A atividade do RF aumentou nas posições para baixo $(27 \%)$ e para frente $(29 \%)$ no ciclista A, e reduziu na posição para baixo (9\%) no ciclista C (Figura 5.2).

O ciclista $B$ apresentou aumento no período de ativação do BF nas posições para baixo (17\%), para cima $(11 \%)$ e para frente $(16 \%)$, conforme ilustra a Figura 5.3; o ciclista A, somente nas posições para baixo (32\%) e para frente (28\%); enquanto que o ciclista $\mathrm{C}$ não registrou diferenças. Para o músculo $\mathrm{VL}$ dos ciclistas (Figura 5.4), constatou-se um aumento no período de atividade nas posições para baixo (15\%) e para frente $(16 \%)$ no ciclista $A$, e na posição para cima (7\%) no ciclista C. O ciclista B não apresentou alteração.

O ciclista A apresentou aumento no período de ativação do GAM, nas posições para baixo (39\%) e para frente $(37 \%)$, em relação à posição de referência; em contrapartida, não foi observada variação para o ciclista C (Figura 5.5). A ativação do músculo TA aumentou nas posições para baixo $(38 \%)$ e para frente $(50 \%)$ no ciclista A, para cima (5\%) no B e em todas as posições no C (para baixo 10\%; para cima 33\%; para frente $19 \%$ e para trás $8 \%)$, diminuindo nas posições para cima (6\%) e para trás (13\%) no ciclista A (Figura 5.6 ).

Para uma melhor compreensão e posterior associação dos resultados de eletromiografia e de cinemetria, um resumo com as mudanças angulares em cada articulação avaliada, nas quatro posições do selim testadas neste estudo, é apresentado na Tabela 3. Os resultados foram normalizados pela geometria articular na posição referência de cada ciclista. Como critério de mudança nos ângulos articulares, adotou-se o mesmo procedimento utilizado para os períodos de ativação, ou seja, valores iguais ou superiores a $5 \%$ em relação à posição de referência individual.

\section{DISCUSSÃO}

O objetivo do presente estudo foi descrever as respostas individuais, no período de ativação muscular, em função de mudanças nas posições vertical e horizontal do selim de ciclistas de elite em relação à posição freqüentemente adotada em treinos e competições (denominada no presente estudo de posição de referência). Comumente são encontrados erros no posicionamento dos atletas em suas bicicletas, e em ciclistas profissionais esses erros geralmente envolvem pequenas dimensões. Testando deslocamentos na ordem de um centímetro para a posição do selim, foram observadas alterações no período de ativação dos músculos monitorados.

No primeiro quadrante, o período de ativação dos músculos GM, RF e VL do ciclista $A$ apresentou aumento tanto na posição com o selim para baixo como quando o selim foi posicionado para frente quando comparado com a posição de referência. Para o ciclista $C$, foi observado aumento do período de ativação apenas do VL na posição para cima; enquanto que os demais músculos, tanto para esse ciclista quanto para o ciclista $B$, não apresentaram diferenças importantes. Os resultados do GM e do RF do ciclista A vão de encontro ao estudo de Desiprés ${ }^{9}$, cujos dados demonstraram maiores períodos de ativação com o selim na posição mais alta, durante a avaliação eletromiográfica de ciclistas competitivos. Porém, para o ciclista C, os achados desse autor estão de acordo no que diz respeito à posição na qual foi registrado maior período de ativação.

No presente estudo, sugere-se que o aumento do período de ativação do $\mathrm{GM}$ do ciclista $\mathrm{A}$, na posição com o selim para baixo, deva-se ao fato de o mesmo partir de um comprimento maior, em função da redução do ângulo do quadril, o que proporciona maior excursão de movimento, já que a ativação do músculo GM está relacionada com o ângulo de flexão do quadril e a existência de resistência para esse movimento ${ }^{23}$.

Ainda em relação ao ciclista $\mathrm{A}$, ocorreu o mesmo com o músculo RF na posição com o selim para frente, ou seja, o período de ativação aumentou, bem como o ângulo da articulação do quadril. Já o ângulo da articulação do joelho reduziu, significando que, teoricamente, o músculo foi ativado em um maior comprimento, fato esse que pode ser explicado por uma mudança na relação força-comprimento, alterando, assim, a estratégia de ativação muscular do ciclista para adaptar-se a essa mudança ${ }^{24}$.

Acredita-se que o aumento no período de ativação do VL do ciclista $A$, nas posições com o selim para baixo e para frente, esteja relacionado com a diminuição do ângulo do joelho, o que pode ocasionar um aumento do comprimento muscular. $O$ fato de esse ciclista estar em uma posição teoricamente menos adequada em relação aquela em que ele está habituado a pedalar e, ainda assim, ter ocorrido aumento da atividade muscular, pode

Tabela 3. Mudanças angulares ocorridas em cada articulação avaliada em relação à posição de referência de cada ciclista ( significa aumento, significa redução dos ângulos articulares, e = significa sem alteração).

\begin{tabular}{|c|c|c|c|c|c|c|c|c|c|c|c|c|}
\hline & \multicolumn{3}{|c|}{ Baixo } & \multicolumn{3}{|c|}{ Cima } & \multicolumn{3}{|c|}{ Frente } & \multicolumn{3}{|c|}{ Trás } \\
\hline & A & B & C & A & B & C & A & B & C & A & B & C \\
\hline Tronco & t & $=$ & $t$ & t & $=$ & $\downarrow$ & $t$ & $=$ & $\downarrow$ & t & - & $\downarrow$ \\
\hline Quadril & $\downarrow$ & t & $\downarrow$ & - & - & - & - & 1 & 1 & $=$ & - & $\downarrow$ \\
\hline Joelho & 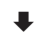 & $\star$ & $\downarrow$ & - & - & 1 & t & $\downarrow$ & 1 & $\downarrow$ & - & $\downarrow$ \\
\hline Tornozelo & t & t & t & $=$ & 1 & $=$ & t & $t$ & t & 1 & - & 1 \\
\hline
\end{tabular}


ser explicado mediante um mecanismo de regulação do sistema nervoso central, que, ciente das condições mecânicas do músculo em termos de comprimento e velocidade, parece enviar maior estímulo ao músculo quando este se encontra em uma situação desfavorável, e o contrário em uma situação mais favoráve ${ }^{25}$.

Durante o segundo quadrante, os ciclistas apresentaram, predominantemente, ativação de GAM; contudo, apenas o ciclista $A$ registrou diferença no período de ativação desse músculo, apresentando maior ativação nas posições com o selim para baixo e para frente. Os resultados do presente estudo vão de encontro aos de Ericson et al..$^{12}$, que apresenta maiores valores de ativação do GAM com o selim na posição para cima. Porém, em ambas as posições, foi observado redução no ângulo da articulação do joelho, o que está associado a mudanças na excursão do músculo GAM e, conseqüentemente, alteração na propriedade força-comprimento.

Com relação ao $\mathrm{BF}$, o aumento no período de ativação dos ciclistas $A$ (para baixo e para frente) e $B$ (para baixo, para cima e para frente) nesse quadrante pode, também, estar associado às alterações na relação força-comprimento como conseqüência das mudanças observadas nos ângulos das articulações do quadril e do joelho. Segundo Mornieux et al. ${ }^{26}$, a altura do selim influencia o pico de momento na articulação do joelho, o que poderia explicar o aumento no período de ativação do BF, quando o selim foi deslocado para baixo e para cima.

Em contrapartida, as mesmas mudanças angulares foram observadas para o ciclista $\mathrm{C}$, porém o mesmo não apresentou mudança nos períodos de ativação em relação à posição de referência. Tal fato pode estar associado a características intrínsecas individuais. Parece não haver um padrão de ativação elétrica, sugerindo que cada ciclista responde de maneira distinta as mudanças no ajuste da posição do selim.

Com relação ao padrão de ativação elétrica no segundo quadrante, os resultados do GAM e BF dos ciclistas avaliados estão de acordo com a literatura revisada. Ao avaliar ciclistas em duas intensidades diferentes, submáxima e máxima ${ }^{27}$, foi observada ativação do GAM entre a metade do primeiro quadrante até o final do segundo, o que está de acordo com o presente estudo para os três ciclistas; e ativação do BF durante o segundo quadrante, o que vai ao encontro das informações dos ciclistas avaliados. É importante destacar ainda, que a ativação do BF é associada ao padrão técnico de pedalada ${ }^{28}$, sendo que este pode mudar entre os ciclistas de elite, o que justificaria as diferenças observadas no período de ativação dos atletas avaliados no presente estudo em relação ao que é reportado na literatura.

O terceiro quadrante foi caracterizado por um maior período de ativação do músculo BF ao longo de todo o ciclo de pedalada, para os três ciclistas, atuando como um flexor do joelho e extensor do quadril. Em um estudo envolvendo triatletas pedalando em duas diferentes inclinações de tronco, 48 e $26^{\circ}$ em relação ao eixo horizontal, e em três diferentes cargas observou-se maior ativação do BF no quarto quadrante, contrariando os resultados deste trabalho ${ }^{29}$. A divergência nos dados pode estar relacionada às distintas estratégias de ativação muscular adotadas por ciclistas e triatletas ${ }^{2}$, assim como pela diferença na ativação descrita anteriormente como decorrência dos diferentes padrões de técnica de pedalada.

Além do BF, também foi observada, no terceiro quadrante, maior ativação do GAM atuando como flexor do joelho. No ciclista A, por um período de tempo maior, enquanto para aos ciclistas B e C somente nos primeiros graus desse quadrante, corroborando os achados de outro estudo que avaliou ciclistas em diferentes alturas de selim ${ }^{3}$.

No quarto quadrante, foi registrado aumento no período de ativação dos músculos RF, VL e TA para os três ciclistas. O RF e o TA apresentaram maior ativação neste quadrante, atuando como flexor do quadril e flexor dorsal, respectivamente; enquanto que o VL foi ativado somente a partir da segunda metade desse quadrante. Nesse quadrante, os ciclistas A e B apresentaram aumento na ativação do RF na posição para baixo, corroborando os resultados encontrados na literatura ${ }^{3,9,10}$. Já para o ciclista $C$ foi registrada uma redução da ativação nesta posição.

Por se tratar de um músculo biarticular, o RF apresentou ativação tanto no quarto (flexor do quadril) como no primeiro (extensor do joelho) quadrantes. $A$ ativação do TA no quarto quadrante representa a técnica do ciclista em direcionar a força no pedal para que esta possa contribuir com o membro contralateral ${ }^{2}$. $\mathrm{Em}$ relação ao $\mathrm{VL}$ os ciclistas $\mathrm{A}$ e $\mathrm{B}$ apresentaram maior ativação no final do quarto quadrante. Sendo este um músculo monoarticular e um potente extensor do joelho ${ }^{13}$, acredita-se que esteja contribuindo para direcionar a força nesse quadrante. Assim, conclui-se que esses atletas detêm melhor técnica ao conseguirem direcionar a força produzida pelo VL no sentido de gerar torque propulsor nesse quadrante.

\section{CONCLUSÃO}

A mudança no ajuste da posição do selim dos ciclistas avaliados afetou os padrões de ativação muscular, fato este que deve estar intimamente ligado a mudanças na técnica de pedalada. Desta forma, pode-se concluir que estes atletas possuem uma adaptação funcional à posição original de treinamento (posição de referência). Acredita-se que os ciclistas adotaram estratégias individuais de adaptação para compensar as mudanças na posição do selim. Embora essas mudanças tenham sido pequenas $(1 \mathrm{~cm})$, os atletas foram sensíveis a elas, talvez por se tratarem de ciclistas de alto rendimento.

\section{REFERÊNCIAS BIBLIOGRÁFICAS}

1. LaFortune MA, Cavanagh PR. Effectiveness and Efficiency during bicycle riding. In: Matsui $\mathrm{H}$, Kobashi K, editors. Biomechanics VIII-B. Champaign, IL: Human Kinetics Publishers; 1983. p. 928-936. 
2. Candotti, CT, Ribeiro J, Soares DP, Oliveira AR, Loss JF, Guimarães ASC. Effective force and economy of triathletes and cyclists. Sport Biomech 2007;6:32-43.

3. Gregor RJ. Biomechanics of cycling. In: Garret WE, Kirkendall DT, editors. Exercise and Sport Science. Philadelphia: Lippincott Williams \& Wilkins; 2000. p. 515-537.

4. Faria, IE. Energy expenditure, aerodynamics and medical problems in cycling. Sports Med 1992;14:4363.

5. Li L, Caldwell GE. Muscle coordination in cycling: effect of surface incline and posture. J Appl Physiol 2003;85(3):927-934.

6. Baum BS, Li L. Lower extremity activities during cycling are influenced by load and frequency. J Eletromyogr Kinesiol 2003;13(2):181-190.

7. GregorRJ, Green D, Garhammer JJ. An electromyographic analysis of selected muscle activity in elite competitive cyclists. In: Biomechanics VII. Baltimore, MD: University Park, 1982. p. 537-541.

8. Houtz, SJ. \& Fischer, F. Analysis of muscle action and joint excursion during exercise on a stationary bicycle. J Bone Joint Surg Am 1959;41:123-131.

9. Desiprés M. An electromyography study of competitive road cycling conditions simulated on a treadmill. In: Nelson RC, Morehouse C, editors. Biomechanics IV. Baltimore, MD: University Park, 1974. p. 349-355.

10. Hull ML, Jorge M. A method for biomechanical analysis of bicycle pedaling. J Biomech 1985;18:631-644.

11. Marsh AP, Martin PE. The relationship between cadence and lower extremity EMG in cyclists and noncyclists. Med Sci Sports Exerc 1995;27(2):217-225.

12. Ericson MO, Nisel R, Arborelius UP, Ekholm J. Muscular activity during ergometer cycling. Scand J Rehabil Med 1985;17(2):53-61.

13. Bini RR, Carpes FP, Diefenthaeler F, MOTA CB, Guimarães ACS. Physiological and electromyographic responses during 40-km cycling time trial: Relationship to muscle coordination and performance J Sci Med Sport 2007 ; in press.

14. Solderberg GL, Knuston L. A guide for use and interpretation of kinesiologic electromyographic data. Physical Therapy 2000;8(5):485-498.

15. Nigg BM, Herzog W. Biomechanics of the muscleskeletal system. Toronto: John Wiley \& Sons; 1994.

16. Merletti R. Standards for reporting EMG data. J Electromyogr Kinesiol 1997;7(2):1-11.

17. Diefenthaeler F, Bini RR, Nabinger E, Laitano OL Carpes, FP, Guimarães ACS, et al. Proposta metodológica para a avaliação da técnica da pedalada de ciclistas: estudo de caso. Rev Bras Med Esporte 2007; in press.

18. Neptune RR, Kautz AS, Hull ML. The effect of pedaling rate on coordination in cycling. J. Biomech 1997;30(10):1051-1058.

19. Ervilha RM, Amadio AC, Duarte M. Estudo sobre procedimentos de normalização da intensidade do sinal eletromiográfico durante o movimento humano. Anais do VII Congresso Brasileiro de Biomecânica. Campinas: UNICAMP; 1997. p.169-174.

20. Moseley L, Jeukendrup AE. The reliability of cycling efficiency. Med Sci Sports Exerc 2001;33(4):621-627.

21. Figueira TR, Denadai BS. Relações entre limiar anaeróbio, limiar anaeróbio individual e máxima fase estável de lactato em ciclistas. R Bras Cie Mov 2004;12(2):91-95.

22. American College of Sports Medicine. Diretrizes do ACSM para os testes de esforço e sua prescrição. 6 ed. Rio de Janeiro: Guanabara Koogan; 2003.

23. Basmajian JV, Deluca CJ. Muscles Alive. Baltimore: Williams \& Wilkins; 1985.

24. Savelberg HC, Van De Port IG, Willems PJ. Body configuration in cycling affects muscle recruitment and movement pattern. J Appl Biomech 2003;19(4):310324.

25. Guimarães AC, Herzog W, Alliger TL, Zhang YT. The EMG-force relationship of the cat soleus muscle and its association with contractile conditions during locomotion. J Exp Biol 1995;198(4):975-987.

26. Mornieux G, Guenette JA, Sheel AW, Sanderson DJ. Influence of cadence, power output and hypoxia on the jointmoment distribution during cycling. Eur J Appl Physiol 2007, in press.

27. Clarys JP, Cabri J, Gregor RJ. The muscle activity paradox during circular rhythmic leg movements. J Sports Sci 1988;6(3):229-237.

28. Duc S; Betik AC, Grappe F. EMG activity does not change during a time trial in competitive cyclists. Int. J. Sport. Med 2005;26:145-150.

29. Carmo, J. Análise eletromiográfica da atual posição de ciclismo. Anais do IV Congresso Brasileiro de Biomecânica. São Paulo: USP; 1992. p.172-178.

\section{Endereço para correspondência}

Fernando Diefenthaeler

Escola de Educação Física - Laboratório de Pesquisa do Exercício

Universidade Federal do Rio Grande do Sul

Recebido em 08/10/07

Revisado em 12/12/07

Rua Felizardo, 750, sala 212, Jardim Botânico

Aprovado em 31/01/08

CEP 90690-200 - Porto Alegre, RS, Brasil

E-mail: fdiefenthaeler@gmail.com 